\title{
Overexpression of IL-9 induced by STAT3 phosphorylation is mediated by miR-155 and miR-21 in chronic lymphocytic leukemia
}

\author{
NA CHEN ${ }^{1}$, LILI FENG ${ }^{1}$, HUITING QU $^{1}, \mathrm{KANG} \mathrm{LU}^{1}$, PEIPEI LI ${ }^{1}, \mathrm{XIAO} \mathrm{LV}^{1}$ and XIN WANG ${ }^{1,2}$ \\ ${ }^{1}$ Department of Hematology, Provincial Hospital Affiliated to Shandong University, Jinan, Shandong 250021; \\ ${ }^{2}$ Institute of Diagnostics, School of Medicine, Shandong University, Jinan, Shandong 250012, P.R. China
}

Received October 22, 2017; Accepted April 3, 2018

DOI: $10.3892 /$ or.2018.6367

\begin{abstract}
Interleukin-9 (IL-9) can function as both a positive and negative regulator of immune response, however the role of IL-9 in tumor immunity is poorly understood. Chronic lymphocytic leukemia (CLL) is the most common chronic lympho-proliferative disorder. Twenty CLL patients from 2010 to 2011 were recruited in the study. Expression and phosphorylation of transcription factor STAT3 and differential microRNAs (miRs) in peripheral blood mononuclear cells (PBMCs) from CLL patient samples were analyzed. In a previous study, we found a high level of IL-9 in CLL patients. Concomitantly, overexpression of pSTAT3, miR-155, and miR-21 were observed in PBMCs from CLL patients in the present study. To elucidate whether there was interaction among IL-9, STAT3, miR-155, and miR-21, MEC-1 cells were used for further study. Our results revealed that there was no detectable IL-9 in the culture medium of MEC-1 cells. However, the IL-9 protein could be detected using western blotting in MEC-1 cells. Notably, when recombinant human IL-9 (rIL-9) was added to the medium of culturing MEC-1 cells, the expression levels of pSTAT3 and IL-9 in MEC-1 cells were increased in a time-dependent manner, which could be blocked by STAT3 inhibitor. Both miR-155 and miR-21 could increase IL-9 expression, which could also be
\end{abstract}

Correspondence to: Professor Xin Wang, Department of Hematology, Provincial Hospital Affiliated to Shandong University, 324 Jingwu Road, Jinan, Shandong 250021, P.R. China

E-mail: xinw007@126.com

Abbreviations: CLL, chronic lymphocytic leukemia; pSTAT, phosphorylation of signal transducer and activator of transcription; miRs, microRNAs; PBMCs, peripheral blood mononuclear cells; rIL-9, recombinant human IL-9; FITC, fluorescein isothiocyanate; PI, Annexin V and propidium iodide; ATL, adult T-cell leukemia; HD, Hodgkin's disease; ALCL, anaplastic large cell lymphoma; NKT, natural killer T cells

Key words: IL-9, CLL, STAT3, miR-155, miR-21 suppressed by the inhibitor of STAT3. Our data indicated that the existence of the novel 'extracellular IL-9/pSTAT3/ miR-155/miR-21/intracellular IL-9' positive feedback system in CLL cells, provides a novel insight in the pathogenesis and possible therapeutic strategy of CLL.

\section{Introduction}

Chronic lymphocytic leukemia (CLL) remains an incurable disease with an extremely variable course $(1,2)$. It is featured by a dynamic imbalance between the proliferation and apoptosis of leukemia cells and by the accumulation of neoplastic B lymphocytes co-expressing CD5 and CD19 antigens (3-6). However, the underlying mechanism involved in the development of this disease still remains unrevealed. In recent years, the role of interleukin (IL)-9 in tumor immunity has obtained increasing attention.

The cytokine IL-9 has largely been regarded as a Th2 cytokine that produces multifocal contributions to allergic disease (7). Recent data revealed that IL-9 is involved in tumor immunity mediated by Treg cells and mast cells (8). Its role in cell growth and the anti-apoptotic process on multiple transformed cells also suggests its effect in tumor progression. IL-9 has also been revealed to participate in IL-2, $-4,-7,-15$ and -21 signaling pathways. These pathways are mediated by heterodimeric receptors that form a specific receptor chain with the common $\gamma$-chain members of the STATs family (9). STAT3 is a latent cytoplasmic transcription factor, originally discovered as a transducer of signals from cell surface receptors to the nucleus. Compelling evidence suggests that STAT3 is constitutively activated in many cancers and plays a pivotal role in tumor growth and metastasis. In addition, STAT3 regulates cellular proliferation, invasion, migration, and angiogenesis, which are critical for cancer metastasis (10).

MicroRNAs are considerably valuable indicators for predicting the clinical behavior of CLL. Structurally, miRNAs are short (19- to 25-nucleotide) RNAs, processed from hairpin loop structures (pre-miRNAs; 60-110 nucleotides in length) that regulate the expression of protein-coding genes as a result of imperfect complementarity with targeting messenger RNAs (11). Several chromosomal abnormalities, such as $11 \mathrm{q}-, 13 \mathrm{q}-, 17 \mathrm{p}-$ and trisomy 12 , and molecular aberrations, 
including loss or downregulation of miRNA-15a and -16-1and overexpression of anti-apoptotic genes, have been identified in CLL in recent years (12). In patients with various $\operatorname{IgVH}$ and ZAP-70 kinase statuses a unique miRNA signature was found to be differentially expressed, and the miRNA signature was composed of the most frequently dysregulated miRNAs in different hematological malignancies (such as miR-15/16, the miR-29 family and miR-155). Recent studies have revealed that miR-21 expression stratifiesthe survival of CLL patients with $17 \mathrm{p}$ - as well as patients with various chromosomal aberrations, suggesting that miR-21 expression could predict patient survival.

Since IL-9, STAT3 and microRNAs are implicated in tumor growth, the cross-talk pathways among these important factors were examined. In the present study, we demonstrated that expression of the pSTAT3 protein, miR-155 and miR-21 were upregulated. Our results revealed that there was a novel 'extracellular IL-9/STAT3/miR-155/miR-21/intracellular IL-9' positive feedback system in CLL cells.

\section{Materials and methods}

Patients and samples. Peripheral blood mononuclear cells (PBMCs) were isolated from heparinized blood obtained from 20 CLL patients who were diagnosed with CLL for the first time at the Department of Hematology, Shandong Provincial Hospital, from January 2010 to December 2011. Samples from 10 healthy volunteers served as normal controls. All patients had not received any treatment, and their lymphocytes exceeded $90 \%$. Density gradient centrifugation was used to isolate the PBMCs from 10 healthy blood donors, and the PBMCs were subjected to a preliminary phenotypic characterization.

Negative selection with antibody-coated magnetic beads was used to obtain a $>97 \%$ pure $\mathrm{CD} 19^{+}$B-cell population, when residual non-B cells exceeded $10 \%$.

The protocol was approved by the Shandong Provincial Hospital Ethics Committee, and written informed consent was obtained from all participants involved in this study.

Cell culture. The human CLL cell line MEC-1 was purchased from the American Type Culture Collection (ATCC; Manassas, VA,USA) and cultured at $37^{\circ} \mathrm{C}$ in $5 \%$ carbon dioxide. Fetal bovine serum (FBS; 10\%) was added to Iscove's modified Dulbecco's medium (IMDM; both from HyClone; GE Healthcare Life Sciences, Logan, UT, USA) to culture the cells.

Transfection of cells. MEC-1 cells $\left(2 \times 10^{5}\right.$ cells/well) were seeded in 24-well plates and incubated overnightand thenwere transfected with the microRNA human miR-155, miR-21 and their negative control miRNAs using Lipofectamine 2000 (Invitrogen; Thermo Fisher Scientific, Inc., Shanghai, China) for $48 \mathrm{~h}$ according to the manufacturer's instructions. The sequences were as follows: miR-155 mimic sense, UUAAUG CUAAUCGUGAUAGGGGU and antisense, CCCUAUCACG AUUAGCAUUAAUU; miR-21 mimic sense, UAGCUUAUCA GACUGAUGUUGA and antisense, AACAUCAGUCUGAU AAGCUAUU; and mimic negative control sense, 5'-UUCUCC GAACGUGUCACGUTT-3' and antisense, 5'-ACGUGACAC GUUCGGAGAATT-3'.
Knockdown of human STAT3 by RNA interference (RNAi). The RNAi target sequence to the human STAT3 gene was: forward, 5'-AAGCAGCAGCTGAACAACATGTTCAAGAGACATGTT GTTCAGCTGCTGCTT-3' and reverse, 5'-AAGCAGCAGC TGAACAACATGTCTCTTGAACATGTTGTTCAGCTGCTG CTT-3'. The sequences of small interfering RNA (siRNA) targeting the human STAT3 gene and negative control siRNA were cloned into the pGCsi.U6/neoRFP plasmid and generated to generate pGC-siSTAT3 by Sangon Biotech Co.,Ltd.(Shanghai, China). As a negative control, a control-siRNA oligonucleotide duplex targeting no known human genes was used: forward, 5'-CCGGTTCTCCGAACGTGTCACGTTTCAAGAGAACGT GACACGTTCGGAGAATTTTTG-3' and reverse, 5'-AATTCA AAAATTCTCCGAACGTGTCACGTTCTCTTGAAACGTG ACACGTTCGGAGAA-3'. pGC-siSTAT3 $(0.8 \mu \mathrm{g})$ and pGC-siCtrl plasmids $(0.8 \mu \mathrm{g})$ were separately transfected into MEC-1 cells $\left(2 \times 10^{5}\right.$ cells/well, 24-well plates $)$ using Lipofectamine 2000 (Invitrogen; Thermo Fisher Scientific, Inc., Shanghai, China) $(2.0 \mu \mathrm{l})$ following the manufacturer's instructions for $4 \mathrm{~h}$, after which time the transfection medium was replaced with regular growth medium. Cells were treated as indicated at $24 \mathrm{~h}$ after transfection.

Co-treatment or co-culture experiments. To explore the effects of extracellular IL-9 on the expression of pSTAT3 and intracellular IL-9 in MEC-1cells, recombinant human IL-9 (rIL-9) was added into the medium. The final concentration of rIL-9 was $20 \mathrm{ng} / \mathrm{ml}$. For STAT3 inhibition, MEC-1 cells were pretreated with WP1066 (Santa Cruz Biotechnology, Inc., Dallas, TX, USA) for $48 \mathrm{~h}$ and then were incubated with rIL-9. The expression level of pSTAT3 and intracellular IL-9 was assessed by western blotting after cells were co-cultured with rIL-9 for 0, 5, 15, 30, 60 and $120 \mathrm{~min}$.

Toelucidate the effects of miR-155 and miR-21 on intracellular IL-9 production, MEC-1 cells and miR-155/miR-21-transfected MEC-1 cells were stimulated with or without WP1066 for $48 \mathrm{~h}$. Cells were then collected, and the expression of the IL-9 protein and mRNA were assessed.

Real-time quantitative polymerase chain reaction (RT-qPCR). Total RNA was extracted from PBMCs of CLL patients and MEC-1 cell lines using TRIzol reagent (Invitrogen; Thermo Fisher Scientific, Inc.) according to the manufacturer's instructions. The reverse transcription reaction and RT-qPCR analysis were performed as previously described (15). Specific primers were bought from Biosune (Shanghai, China), and the primer sequences are shown in Table I.

Western blot analysis. Total protein was extracted from PBMCs of CLL patients and MEC-1 cells and B cells of healthy samples. The determination of protein concentrations and detailed procedures of the immunoblot analysis have been previously described (16). The antibody of GAPDH (1:1,000; cat. no. sc-47724) was purchased from Santa Cruz Biotechnology, while IL-9 (1:10,000; cat. no. B02925) was obtained from Wuhan Boster Biological Technology, Ltd. (Wuhan, China). All other antibodies, STAT3 antibody (1:10,000; cat. no. ab76315) and pSTAT3 antibody $(1: 1,000$; cat. no. ab32500) were purchased from Abcam (Cambridge, MA, USA). 
Table I. The primer sequences.

\begin{tabular}{ll}
\hline Gene Name & \multicolumn{1}{c}{ Sequence } \\
\hline IL-9 & 5'-CTCTGTTTGGGCATTCCCTCT-3' \\
& 5'-GGGTATCTTGTTTGCATGGTGG-3' \\
miR-155 & 5'-TTAATGCTAATCGTGA-3' \\
& 5'-TTTGGCACTAGCACATT-3' \\
miR-21 & 5'-TAGCTTATCAGACTGATG-3' \\
& 5'-TTTGGCACTAGCACATT-3' \\
3-actin & 5'-CATTAAGGAGAAGCTGTGCT-3' \\
& 5'-GTTGAAGGTAGTTTCGTGGA-3' \\
U6 & 5'-CTCGCTTCGGCAGCACA-3' \\
& 5'-AACGCTTCACGAATTTGCGT-3'
\end{tabular}

Assessment of cell proliferation and apoptosis. Both transfected MEC-1 cells and untransfected MEC-1 cells were seeded into 96 -well plates at a concentration of $5 \times 10^{3}$ cells/well. The cells were treated with or without WP1066 (10 nM) for $48 \mathrm{~h}$ previously to seeding.

Cells were then incubated with rIL-9 $(20 \mathrm{ng} / \mathrm{ml})$ for 0,15 , $30,60,90$ and $120 \mathrm{~min}$. At the indicated time-points, cell proliferation was evaluated using Cell Counting Kit-8 (Beyotime Institute of Biotechnology, Haimen, China) according to the manufacturer's instructions. After cells were incubated with rIL-9 $(20 \mathrm{ng} / \mathrm{ml})$ for $120 \mathrm{~min}$, fluorescein isothiocyanate (FITC)-Annexin V and propidium iodide (PI) were used to analyze apoptotic and necrotic cells (Neobioscience Co., Ltd., Shenzhen, China). Briefly, Annexin V-FITC and PI were used to incubate cells $\left(10^{6}\right)$ for $10 \mathrm{~min}$ in the dark at room temperature. Cells were then immediately analyzed with FACS can flow cytometer (Beckman Coulter, Chicago, IL, USA). Viable cells are not stained. The necrotic cells were Annexin V-FITC and PI-positive, whereas apoptotic cells were Annexin V-FITC-positive and PI-negative.

Statistical analysis. SPSS version 17.0 (SPSS, Inc., Chicago, IL, USA) for Windows was used to perform statistical analyses. The numerical data were statistically analyzed using the two-tailed Student's t-test. $\mathrm{P}<0.05$ was considered to indicate a statistically significant difference.

\section{Results}

Expression of pSTAT3, miR-155 and miR-21 in CLL patients. An elevated level of IL-9 was detected in sera from 20 out of 47CLL patients in our previous study $(17,18)$. Expression of STAT3 and pSTAT3 in PBMCs from the 20 CLL patients with upregulated IL-9 and 10 healthy controls was detected using western blotting. As shown in Fig. 1A and B, the expression of pSTAT3 was higher in CLL patients than that in healthy controls.

Expression of miR-155 and miR-21 in the 20 CLL patients and 10 healthy controls was assessed using RT-qPCR. We found that miR-155 and miR-21 were elevated in CLL samples compared with the controls (Fig. 1C and D).
rIL-9 increases IL-9 expression through phosphorylation of STAT3 in MEC-1 cells. The expression of IL-9 in the medium of MEC-1 cells was assessed and no IL-9 was detected. Then, rIL-9 was added to stimulate MEC-1 cells. The expression of pSTAT3 and IL-9 are shown in Fig. 2A and the results revealed that the expression of pSTAT3 and IL-9 increased in a time-dependent manner.

Furthermore, this increase could be suppressed by Wp1066, which is a STAT3 inhibitor (Fig. 2B and C). Expression of pSTAT3 and IL-9 were detected by western blotting, with the single band size of 88 and $14 \mathrm{kDa}$, respectively, in peripheral blood mononuclear cells (PBMCs) from healthy samples and human CLL cell line MEC-1, after co-culture with rIL-9 for 120 min. pSTAT3 and IL-9 protein levels were almost undetectable in PBMCs from healthy samples (Fig. 2D).

To further explore the potential association between IL-9 and pSTAT3 in CLL, the IL-9 protein was evaluated in STAT3-depleted cells after co-culture with rIL-9 for 120 min (Fig. 2E). As shown in Fig. 2F, STAT3 knockdown reduced IL-9 expression $(\mathrm{P}<0.0001)$. These results indicated that rIL-9 (extracellular IL-9) could stimulate MEC-1 cells to produce IL-9 through the phosphorylation of STAT3.

STAT3 phosphorylation regulates IL-9 production and is associated with miR-155 and $m i R-21$. Since the phenomenon that activated STAT3 has a marked effect on the upregulation of miR-155 and miR-21 expression in CLL cells (19), we further explored whether IL-9 levels would be affected by miR-155 and miR-21 in CLL cells. After transfection with miR-155 and miR-21 for $48 \mathrm{~h}$, rIL-9 $(20 \mathrm{ng} / \mathrm{ml})$ was used to stimulate MEC-1 cells for $120 \mathrm{~min}$ and then harvested to determine the expression of IL-9 using western blotting. We determined that IL-9 expression was increased in transfected cells when compared with untransfected ones and negative controls. When miR-transfected MEC-1 cells were pretreated with WP1066 for 48 h, WP1066 could suppress IL-9 production in cells treated with rIL-9. The protein level of IL-9 was higher in miR-155 and miR-21 transfected cells compared to miR-155 or miR-21 transfected cells, but there was no statistical significance (Fig. 3A and B). In addition, we demonstrated that overexpression of miR-155 and miR-21 could promote the production of IL-9 in CLL cells. As shown in Fig. 3C and D, rIL-9 treatment promoted time-dependent increase in the expression of miR-155 and miR-21, which was significantly blocked by WP1066 (Fig. 3E and F). Given the upregulation of pSTAT3 to miR-155 and miR-21 expression and the upregulation of miR-155 and miR-21 to IL-9 production in CLL cells, it appeared that the upregulation of IL-9 regulated by STAT3 phosphorylation was mediated by miR-155 and miR-21.

IL-9 promotes MEC-1 cell proliferation and inhibits apoptosis. To determine the effect of extracellular IL-9 on the cell growth of MEC-1cells, we first assayed its effect on the proliferation of these cells. As shown in Fig. 4A, rIL-9 could promote MEC-1 cell proliferation after co-culture for $30 \mathrm{~min}$. However, pretreatment with WP1066 could significantly suppress the proliferative effects of rIL-9 on MEC-1 cells. Moreover, the proliferative effects of rIL-9 on miR-155- or miR-21-transfected MEC-1 cells were more apparent than 


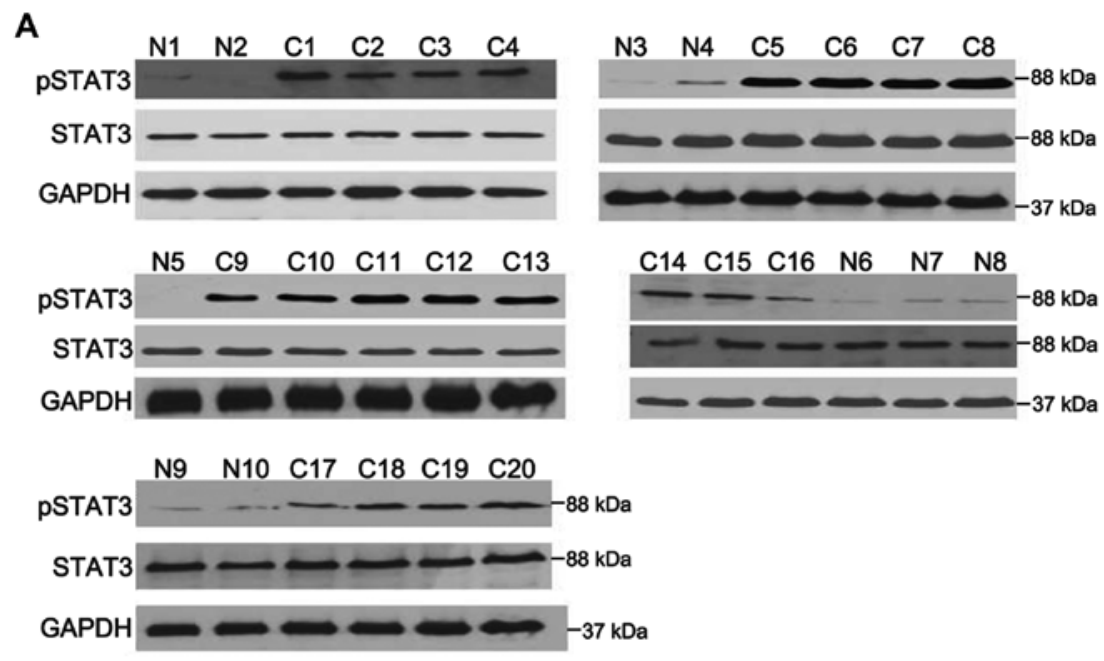

B

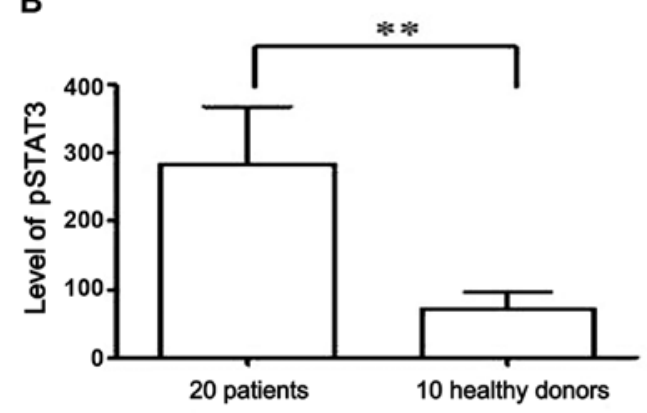

D

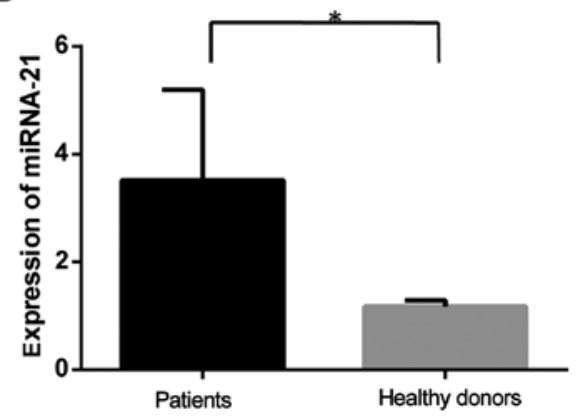

C

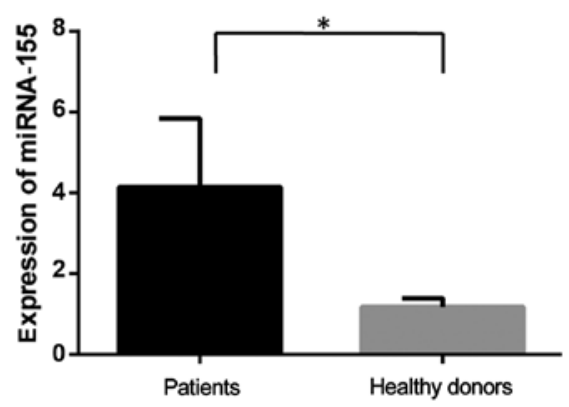

Figure 1. Expression of pSTAT3 protein and miRNAs in CLL patients and healthy controls. (A) The expression of the pSTAT3 protein was detected in 20 samples with upregulation of IL-9 (C), but none was detected in 10 PBMC from normal controls (N). (B) Relative density analysis of pSTAT3 protein bands. ${ }^{* *} \mathrm{P}<0.0001$. (C) RT-qPCR analysis of miR-155 expression in CLL patients and healthy controls. " $\mathrm{P}=0.0308$. (D) RT-qPCR analysis of miR-21 expression in patient samples and healthy donors. ${ }^{*} \mathrm{P}=0.0398$.

MEC-1 cells. Pretreatment with WP1066 could also suppress the proliferative effects of rIL-9 on transfected MEC-1 cells (Fig. 4B).

In addition to the promotion of cell proliferation, other mechanisms could also participate in the regulation of IL-9-mediated MEC-1 cell growth. In our following experiments, we examined the effects of rIL-9 on apoptosis and necrosis of CLL. As shown in Fig. 4C and E, rIL-9 could reduce cell apoptosis to $\sim 60 \%$ of the baseline level. Pretreatment with WP1066 could significantly abolish the anti-apoptotic effects of rIL-9 on MEC-1 cells. When cultured without rIL-9, the apoptosis rate of miR-155/miR-21-transfected MEC-1 cells was lower than that in MEC-1 cells and negative controls, which demonstrated that IL-9 produced by CLL cells itself could also inhibit CLL cell apoptosis. Pretreatment with WP1066 could abolish the anti-apoptotic effects of rIL-9 on transfected MEC-1 cells (Fig. 4D and F).

\section{Discussion}

In hematological malignancies, although aberrant expression of IL-9 has been observed, the role of IL-9 in the pathological significance of CLL has not been reported. In our previous study, the results revealed an elevated level of IL-9 in CLL patients. Upregulation of serum IL-9 is significantly associated with the clinical stage, ZAP-70, B2M and IgVH in CLL patients $(17,18)$. In the present study, high expression of pSTAT3, miR-155 and miR-21 were observed in PBMCs 


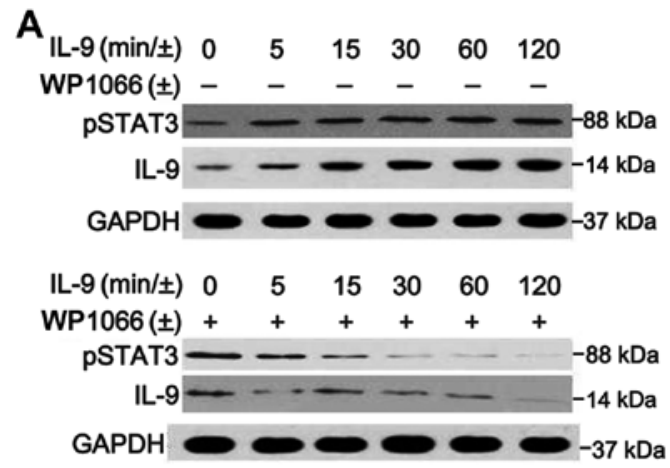

C

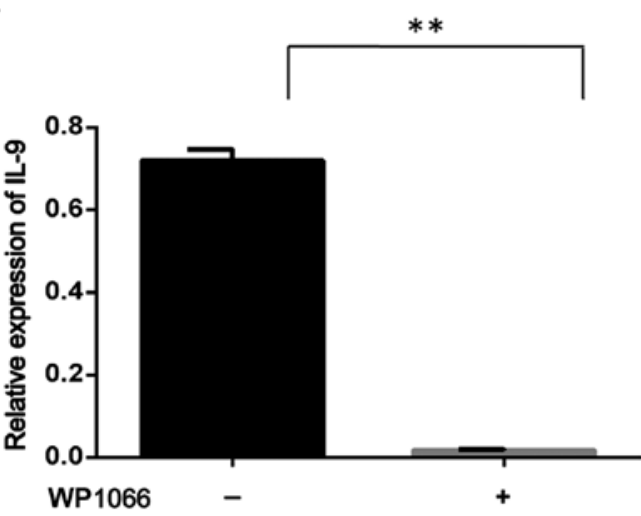

E

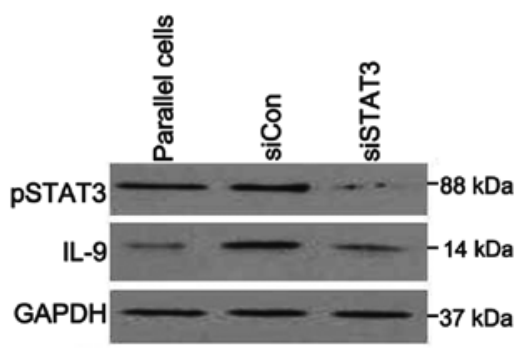

B

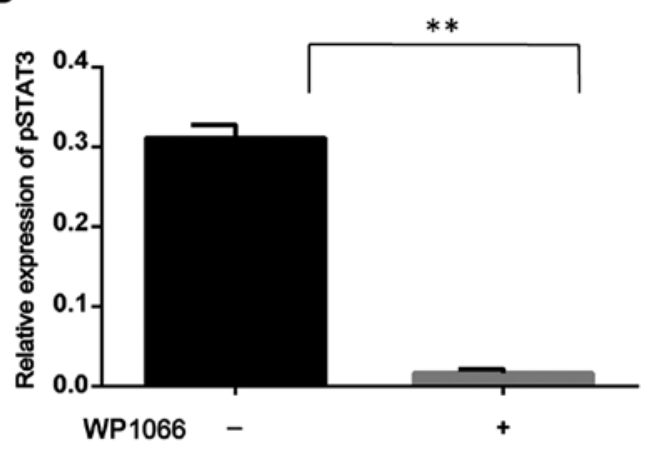

D

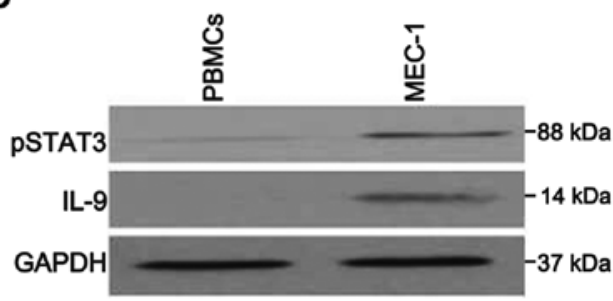

Figure 2. Effects of rIL-9 on pSTAT3 and IL-9 production in MEC-1 cells.(A) MEC-1 cells were pretreated with or without WP1066 for 48 h and then were incubated with rIL-9 for different times. STAT3 phosphorylation and IL-9 production in MEC-1 cells increased with the stimulation of rIL-9 in a time-dependent manner. WP1066, the STAT3 inhibitor, could markedly suppress the effects of rIL-9 on MEC-1 cells. (B) Relative density analysis of pSTAT3 protein bands, which were cultured with rIL-9 for $120 \mathrm{~min} .{ }^{* *} \mathrm{P}<0.0001$. (C) Relative density analysis of IL-9 protein bands, which were treated with rIL-9 for 120 min ${ }^{* *} \mathrm{P}<0.0001$. (D) Expression of pSTAT3 and IL-9 were detected by western blotting, with the single band size of 88 and $14 \mathrm{kDa}$, in peripheral blood mononuclear cells (PBMCs) from healthy samples and human CLL cell line MEC-1 after co-culture with rIL-9 for 120 min. (E and F) STAT3-knockdown cells exhibited significantly lower IL-9 expression in comparison to the cells transfected with negative control siRNA after co-culture with rIL-9 for 120 min. ${ }^{* *} \mathrm{P}<0.0001$.

from CLL patients. Furthermore, there was a complex link among IL-9, STAT3, miR-155 and miR-21. Collectively, our results indicated novel mechanisms involved in CLL pathology: i) extracellular IL-9 could promote the generation of IL-9 in CLL cells; and ii) there is a novel 'extracellular IL-9/pSTAT3/miR-155/miR-21/intracellular IL-9' positive feedback system in CLL cells.

IL-9 is a member of the common $\gamma$-chain family of cytokines, and the IL-9receptor consists of the combination of the cytokine-specific receptor IL-9 receptor- $\alpha$ (IL-9R $\alpha)$ and the $\gamma$-chain (9). In addition to its role in immune responses, its growth and anti-apoptotic activities on multiple transformed cells suggest potential effects in hematological malignancies. The dysregulated expression of IL-9 has been detected in biopsies or serum of patients with some hematological malignancies, such as adult T-cell leukemia (ATL), Hodgkin's disease (HD), an aplastic large cell lymphoma (ALCL), and natural killer T (NKT)-cell lymphoma. All these studies provided evidence that IL-9 plays an important role in pathogenesis of hematological malignancies (20-22). Our previous study indicated that IL-9 could contribute to CLL progression $(17,18)$. However, the pathogenic role of IL-9 in CLL remains largely unknown.

Research findings from cell culture and animal models have revealed that the Jak/STAT signaling pathway, which can be activated by many cytokines, including IL-9, plays a critical role in hematological malignancies $(23,24)$. STAT3 mutations are consistent with the pathogenesis of chronic 
A

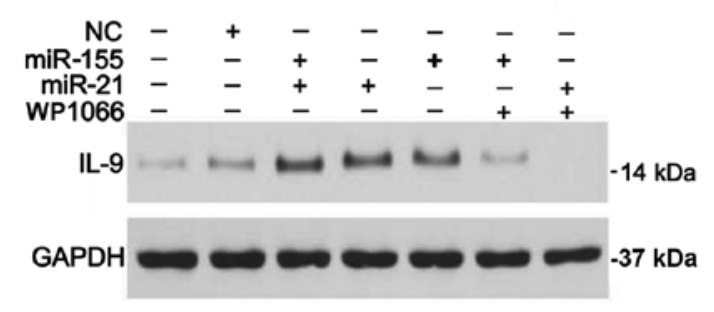

C

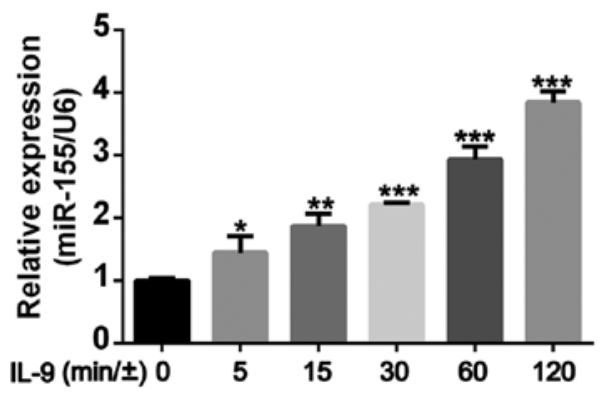

E

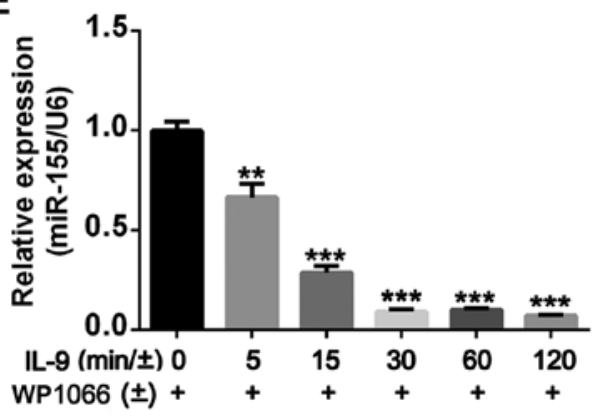

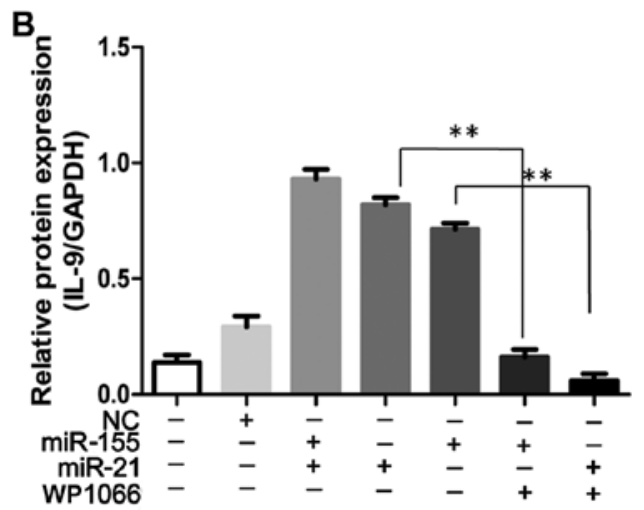

D

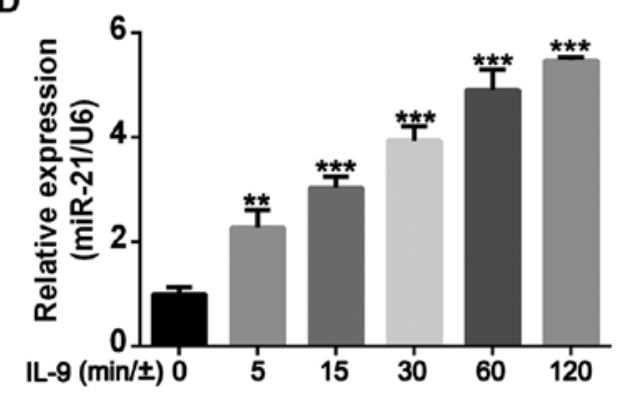

$\mathbf{F}$

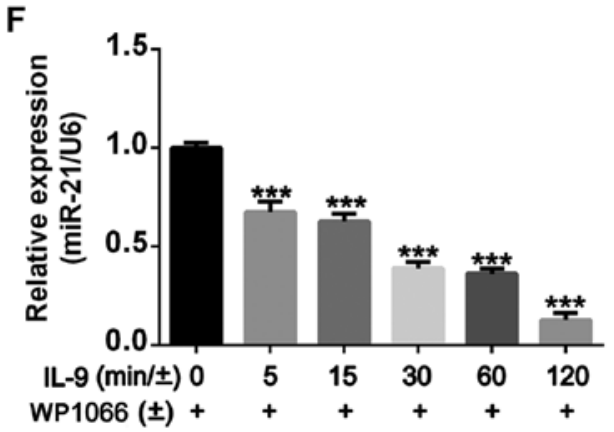

Figure 3. STAT3 phosphorylation regulatesIL-9 production, and is associated with miR-155 and miR-21. (A) MEC-1 cells, negative control cells and miRNAtransfected CLL cells were stimulated with $20 \mathrm{ng} / \mathrm{ml}$ rIL-9 for $120 \mathrm{~min}$ followed by treatment with WP1066 (10 nM) for $48 \mathrm{~h}$. Western blot analysis was used to detect the IL-9 protein levels in these groups. GAPDH was loaded as a control. (B) Relative density analysis of IL-9 protein bands in MEC-1 cells, negative control cells and miRNA-transfected MEC-1 cells. ${ }^{* *} \mathrm{P}<0.0001$. (C-F) MEC-1 cells were pretreated with or without WP1066 for $48 \mathrm{~h}$ and then were incubated with rIL-9 for different times. Relative expression of miR-155 and miR-21 were determined by $\mathrm{RT}-\mathrm{qPCR}$. ${ }^{*} \mathrm{P}<0.05,{ }^{* * *} \mathrm{P}<0.01$ and ${ }^{* * *} \mathrm{P}<0.001$.

lympho-proliferative disorders of NK cells and T-cell large granular lymphocyte leukemia (25). Mutations were found in exons 20 and 21, encoding the Src homology 2 domain. In acute myeloid leukemia cells, murine plasmacytomas, and hybridomas, the autocrine secretion of interleukin-6 caused phosphorylation of STAT3, Tyr705 and Ser727 $(9,26)$, including STAT3-mediated constitutive expression of SOCS-3 in cutaneous T-cell lymphoma (27). In addition, STAT3 also plays a pivotal role in the progression of hematological malignancies, including CLL (28-30). In the present study, our results revealed that pSTAT3 expression was elevated in PBMCs from CLL patients. Moreover, extracellular rIL-9 could induce STAT3 phosphorylation in the MEC-1 cell line. The rIL-9-mediated pSTAT3 expression may be related to the pathogenic role of CLL. Many studies have shown that the IL-9R $\alpha$ chain promoted the phosphorylation of JAK1 mutant and activation of STAT, including STAT1, STAT3 and STAT5 $(9,31,32)$ and-IL-9 could not be expressed by Th2 and Th9 cells without expression of STAT6 (33). To assess the association between pSTAT3 and IL-9, MEC-1 cells were chosen for our study. We found that extracellular IL-9 could not only promote STAT3 phosphorylation but also increase IL-9 production by MEC-1 cells.

Since miR levels are dysregulated in CLL and STATs are activated in CLL, we attempted to determine whether STATs affect the transcription of miRNA genes in CLL cells. Recently, studies revealed that STAT3 stimulation induced miR-155 and miR-21 upregulation in CLL cells $(15,16)$. In our study, we also demonstrated that miR-155 and miR-21 expression was upregulated in PBMCs from CLL patients. The changes in miR-155 and miR-21 expression were accompanied by a substantial increase of IL-9 expression. Furthermore, blocking STAT3 in miR-155- and miR-21-transfected cells was associated with a reduction of IL-9. These data revealed that IL-9 production induced by pSTAT3 was mediated by miR-155 and miR-21. Since extracellular IL-9 could not only promote STAT3 phosphorylation but also increase IL-9 production in MEC-1 cells, we concluded that there was a novel 'extracellular 
A

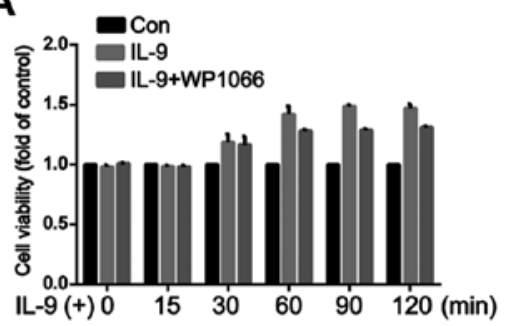

B

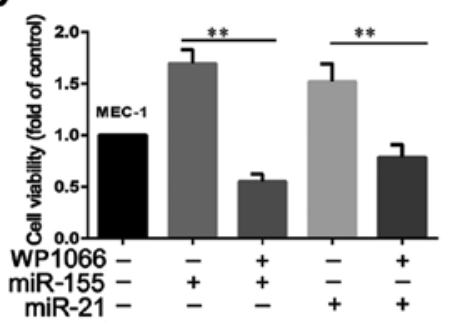

C

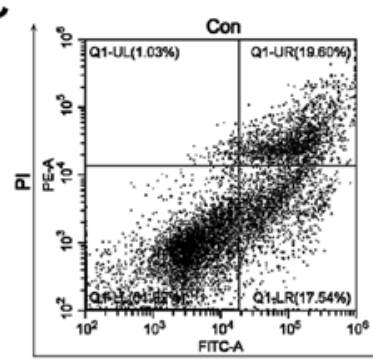

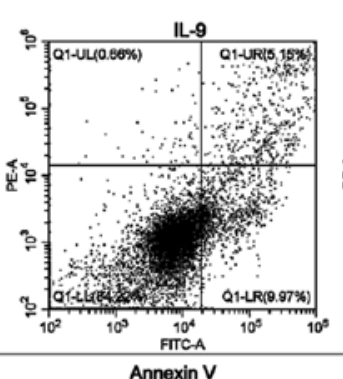

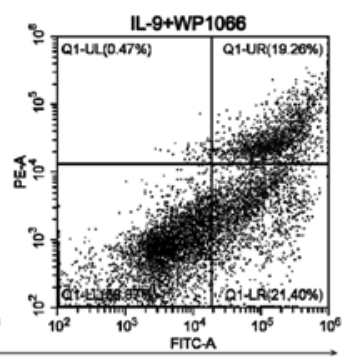

D

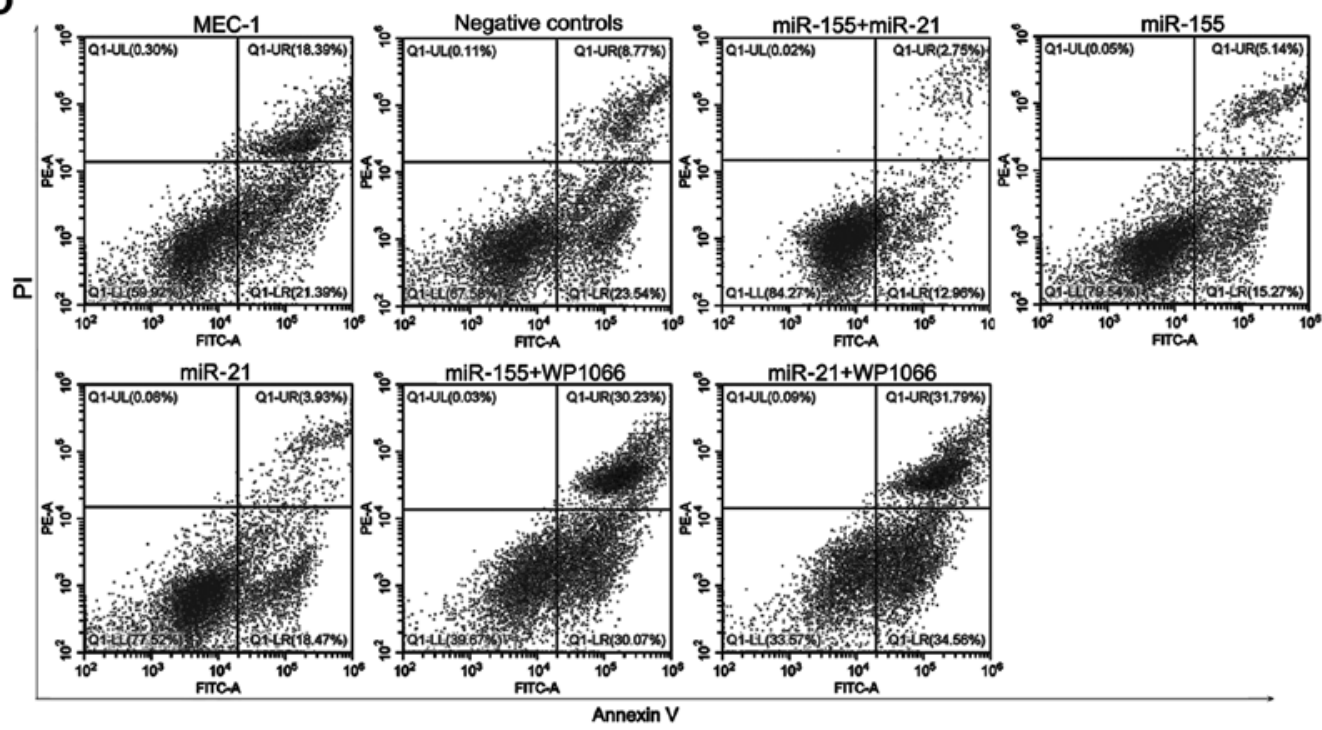

E

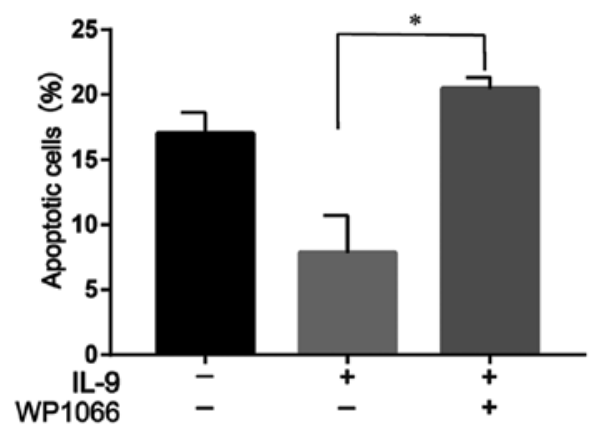

F

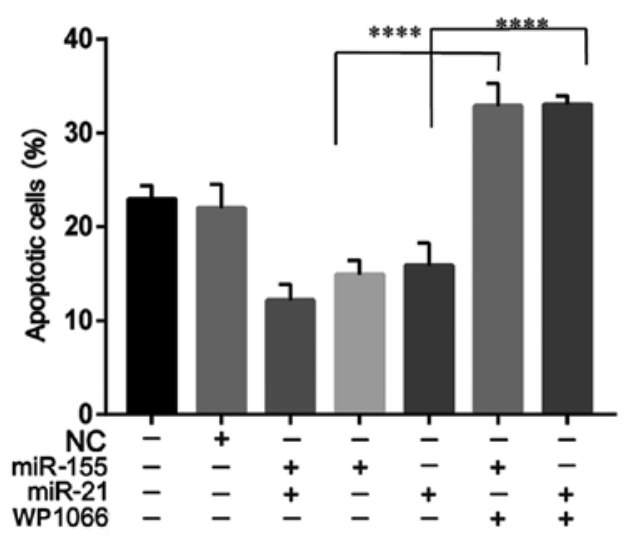

Figure 4. Effects of IL-9 on proliferation and apoptosis of MEC-1 cells. (A) MEC-1 cells were co-cultured with or without WP1066 for $48 \mathrm{~h}$ and then were treated with $20 \mathrm{ng} / \mathrm{ml}$ IL-9 for different times. Then, cell proliferation was evaluated using cell counting kit-8. (B) The miRNA155/miRNA21-transfected cells were co-cultured with or without WP1066 for $48 \mathrm{~h}$ and then treated with $20 \mathrm{ng} / \mathrm{ml}$ IL-9 for $120 \mathrm{~min}$. Cell proliferation was evaluated using cell counting kit-8. ${ }^{* *} \mathrm{P}<0.000$. (C) WP1066 (10 nM) and rIL-9 were used to treat cells successively for $48 \mathrm{~h}$ and $120 \mathrm{~min}$, respectively. An Annexin V-FITC and PI apoptosis detection kit was used to detect cell apoptosis and necrosis. (D) MEC-1 cells and miRNA155/miRNA21-transfected MEC-1 cells received WP1066 (10 nM) treatment for $48 \mathrm{~h}$ and then were collected for apoptosis detection. (E-F) Columns indicate the mean apoptosis rate of triplicate determinations; bars, SD. ${ }^{*} \mathrm{P}<0.05$ and ${ }^{* * * * *} \mathrm{P}<0.0001$. 
IL-9/pSTAT3/miR-155/miR-21-intracellular IL-9' positive feedback system in CLL cells.

To explore the potential function of IL-9 in CLL pathogenesis, we investigated the effects of rIL-9 on the proliferation and apoptosis of CLL cells. After treatment with rIL-9, the proliferation of MEC-1 cells increased, whereas their apoptosis decreased. Also, STAT3 inhibitor could significantly counteract the effects of rIL-9. Moreover, the proliferative effects of rIL-9 on miR-155/miR-21-transfected MEC-1 cells were more apparent than in MEC-1 cells. Although it was difficult to determine whether the rIL-9 or the IL-9 produced by MEC-1 cells played the dominant role in this process, the upregulation of IL-9 may play a role in the pathogenesis of CLL.

Collectively, we found that extracellular IL-9 could not only activate the transcription of the STAT3 gene but also upregulate the expression of miR-155 and miR-21. Consequently, the STAT3-mediated expression of miR-155 and miR-21 promoted IL-9 secretion. Our findings provide a new explanation of the possible molecular mechanism in the regulation of IL-9 in CLL. It may provide valuable insights in understanding the cross-talk pathways among IL-9, STAT3, miR-155 and miR-21, which may play a key role in the pathogenesis of CLL.

The data we present here explored the cross-talk pathways among IL-9, STAT3, miR-155 and miR-21 in CLL. Indeed, recent research has shown that IL-9 could contribute to CLL progression. Our study provides evidence that the expression of pSTAT3, miR-155 and miR-21 was increased in CLL with IL-9 upregulation. The STAT3 phosphorylation in MEC-1 cells stimulated by rIL- 9 was associated with a time-dependent increase in IL-9 secretion. Pretreatment with WP1066 eliminated the IL-9 secretion despite the presence of rIL-9. IL-9 protein levels were increased in miR-155-transfected MEC-1 cells and miR-21-transfected MEC-1 cells treated with rIL-9 at 120 min compared with untransfected cells, which could be blocked by WP1066. In transfected MEC-1 cells apoptosis inhibition and the proliferation enhanced by rIL-9 could be blocked by WP1066. This perspective is particularly intriguing in that extracellular IL-9 appears to not only activate the transcription of the STAT3 gene but also to upregulate miR-155 and miR-21 expression. Consequently, the STAT3-mediated translational upregulation of miR-155 and miR-21 promoted IL-9 secretion.

\section{Acknowledgements}

Not applicable.

\section{Funding}

This study was partly supported by the National Natural Science Foundation (nos. 81270598 and 81500124), the Natural Science Foundations of Shandong Province (nos. Y2007C053, 2009ZRB14176 and ZR2012HZ003), the Technology Development Projects of Shandong Province (nos. 2007GG10 and 2010GSF10250), the Major Research Projects of Shandong Province (no. 2017GSF218007), the Program of Shandong Medical Leading Talent, and the Taishan Scholar Foundation of Shandong Province.

\section{Availability of data and materials}

The analyzed data sets generated during the study are available from the corresponding author upon reasonable request.

\section{Authors' contributions}

$\mathrm{CN}$ and WX conceived and designed the study. CN, FLL and LK performed the experiments. $\mathrm{CN}$ and $\mathrm{LX}$ wrote the paper. $\mathrm{CN}, \mathrm{QHT}, \mathrm{LPP}$ and WX reviewed and edited the manuscript. All authors read and approved the manuscript and agree to be accountable for all aspects of the research in ensuring that the accuracy or integrity of any part of the work are appropriately investigated and resolved.

\section{Ethics approval and consent to participate}

The protocol was approved by the Shandong Provincial Hospital Ethics Committee, and written informed consent was obtained from all participants involved in this study.

\section{Consent for publication}

Not applicable.

\section{Competing interests}

The authors declare that they have no competing interests.

\section{References}

1. Negro R, Gobessi S, Longo PG, He Y, Zhang ZY, Laurenti L and Efremov DG: Overexpression of the autoimmunity-associated phosphatase PTPN22 promotes survival of antigen-stimulated CLL cells by selectively activating AKT. Blood 119: 6278-6287, 2012.

2. Oi T, Asanuma K, Matsumine A, Matsubara T, Nakamura T, Iino T, Asanuma Y, Goto M, Okuno K, Kakimoto T, et al: STAT3 inhibitor, cucurbitacin I, is a novel therapeutic agent for osteosarcoma. Int J Oncol 49: 2275-2284, 2016.

3. Fecteau JF, Messmer D, Zhang S, Cui B, Chen L and Kipps TJ: Impact of oxygen concentration on growth of mesenchymal stromal cells from the marrow of patients with chronic lymphocytic leukemia. Blood 121: 971-974, 2013.

4. Rawstron AC, Böttcher S, Letestu R, Villamor N, Fazi C, Kartsios H, de Tute RM, Shingles J, Ritgen M, Moreno C, et al; European Research Initiative in CLL: Improving efficiency and sensitivity: European Research Initiative in CLL (ERIC) update on the international harmonised approach for flow cytometric residual disease monitoring in CLL. Leukemia 27: 142-149, 2013.

5. Samuel S, Beljanski V, Van Grevenynghe J, Richards S, Ben Yebdri F, He Z, Nichols C, Belgnaoui SM, Steel C, Goulet ML, et al: BCL-2 inhibitors sensitize therapy-resistant chronic lymphocytic leukemia cells to VSV oncolysis. Mol Ther 21: 1413-1423, 2013.

6. Fang C, Zhuang Y, Wang L, Fan L, Wu YJ, Zhang R, Zou ZJ, Zhang LN, Yang S, Xu W, et al: High levels of CD20 expression predict good prognosis in chronic lymphocytic leukemia. Cancer Sci 104: 996-1001, 2013.

7. Soroosh P and Doherty TA: Th9 and allergic disease. Immunology 127: 450-458, 2009.

8. Tete S, Saggini A, Maccauro G, Rosati M, Conti F, Cianchetti E, Tripodi D, Toniato E, Fulcheri M, Salini V, et al: Interleukin-9 and mast cells. J Biol Regul Homeost Agents 26: 319-326, 2012.

9. Hornakova T, Staerk J, Royer Y, Flex E, Tartaglia M, Constantinescu SN, Knoops Land Renauld JC: Acute lymphoblastic leukemia-associated JAK1 mutants activate the Janus kinase/ STAT pathway via interleukin-9 receptor alpha homodimers. J Biol Chem 284: 6773-6781, 2009. 
10. Fionda C, Malgarini G, Soriani A, Zingoni A, Cecere F, Iannitto ML, Ricciardi MR, Federico V, Petrucci MT, Santoni A, et al: Inhibition of glycogen synthase kinase-3 increases NKG2D ligand MICA expression and sensitivity to NK cell-mediated cytotoxicity in multiple myeloma cells: Role of STAT3. J Immunol 190: 6662-6672, 2013

11. Chen DL, Wang DS, Wu WJ, Zeng ZL, Luo HY, Qiu MZ, Ren C, Zhang DS, Wang ZQ, Wang FH, et al: Overexpression of paxillin induced by miR-137 suppression promotes tumor progression and metastasis in colorectal cancer. Carcinogenesis 34: 803-811, 2013

12. Iliopoulos D, Jaeger SA, Hirsch HA, Bulyk ML and Struhl K STAT3 activation of miR-21 and miR-181b-1 via PTEN and CYLD are part of the epigenetic switch linking inflammation to cancer. Mol Cell 39: 493-506, 2010.

13. Nakamura H, Taguchi A, Kawana K, Kawata A, Yoshida M, Fujimoto A, Ogishima J, Sato M, Inoue T, Nishida H, et al: STAT3 activity regulates sensitivity to tumor necrosis factorrelated apoptosis-inducing ligand-induced apoptosis in cervical cancer cells. Int J Oncol 49: 2155-2162, 2016.

14. Bartel DP: MicroRNAs: Target recognition and regulatory functions. Cell 136: 215-233, 2009.

15. Hartman ML and Kilianska ZM: Lipoprotein lipase: A new prognostic factor in chronic lymphocytic leukaemia. Contemp Oncol (Pozn) 16: 474-479, 2012

16. ENCODE Project Consortium: Myers RM, Stamatoyannopoulos J, Snyder M, Dunham I, Hardison RC, Bernstein BE, Gingeras TR, Kent WJ, Birney E, Wold B, et al: A user's guide to the encyclopedia of DNA elements (ENCODE). PLoS Biol 9: e1001046, 2011.

17. Chen N, Lv X, Li P, Lu K and Wang X: Role of high expression of IL-9 in prognosis of CLL. Int J Clin Exp Pathol 7: 716-721, 2014

18. Chen N, Lu K, Li P, Lv X and Wang X: Overexpression of IL-9 induced by STAT6 activation promotes the pathogenesis of chronic lymphocytic leukemia. Int J Clin Exp Pathol 7: 2319-2323, 2014.

19. Barski A, Cuddapah S, Cui K, Roh TY, Schones DE, Wang Z, Wei G, Chepelev I and Zhao K: High-resolution profiling of histone methylations in the human genome. Cell 129: 823-837, 2007.

20. Chen J, Petrus M, Bryant BR, Phuc Nguyen V, Stamer M, Goldman CK, Bamford R, Morris JC, Janik JE and Waldmann TA: Induction of the IL-9 gene by HTLV-I Tax stimulates the spontaneous proliferation of primary adult T-cell leukemia cells by a paracrine mechanism. Blood 111: 5163-5172, 2008.

21. Matsushita K, Arima N, Ohtsubo H, Fujiwara H, Hidaka S, Fukumori J and Tanaka H: Frequent expression of interleukin-9 mRNA and infrequent involvement of interleukin-9 in proliferation of primary adult T-cell leukemia cells and HTLV-I infected T-cell lines. Leuk Res 21: 211-216, 1997.

22. Ju W, Zhang M, Jiang JK, Thomas CJ, Oh U, Bryant BR, Chen J, Sato N, Tagaya Y, Morris JC, et al: CP-690,550, a therapeutic agent, inhibits cytokine-mediated Jak3 activation and proliferation of T cells from patients with ATL and HAM/TSP. Blood 117: 1938-1946, 2011.
23. Schuringa JJ, Wierenga AT, Kruijer W and Vellenga E: Constitutive Stat3, Tyr705, and Ser727 phosphorylation in acute myeloid leukemia cells caused by the autocrine secretion of interleukin-6. Blood 95: 3765-3770, 2000.

24. Brender C, Nielsen M, Kaltoft K, Mikkelsen G, Zhang Q, Wasik M, Billestrup N and Odum N: STAT3-mediated constitutive expression of SOCS-3 in cutaneous T-cell lymphoma. Blood 97: 1056-1062, 2001

25. Steele AJ, Prentice AG, Cwynarski K, Hoffbrand AV, Hart SM, Lowdell MW, Samuel ER and Wickremasinghe RG: The JAK3-selective inhibitor PF-956980 reverses the resistance to cytotoxic agents induced by interleukin- 4 treatment of chronic lymphocytic leukemia cells: Potential for reversal of cytoprotection by the microenvironment. Blood 116: 4569-4577, 2010.

26. Goswami R, Jabeen R, Yagi R, Pham D, Zhu J, Goenka S and Kaplan MH: STAT6-dependent regulation of Th9 development. J Immunol 188: 968-975, 2012.

27. Rozovski U, Calin GA, Setoyama T, D'Abundo L, Harris DM,Li P, Liu Z, Grgurevic S, Ferrajoli A, Faderl S, et al: Signal transducer and activator of transcription (STAT)-3 regulates microRNA gene expression in chronic lymphocytic leukemia cells. Mol Cancer 12: 50, 2013.

28. Li P, Grgurevic S, Liu Z, Harris D, Rozovski U, Calin GA, Keating MJ and Estrov Z: Signal transducer and activator of transcription-3 induces microRNA-155 expression in chronic lymphocytic leukemia. PLoS One 8: e64678, 2013.

29. Shaim H, Estrov Z, Harris D, Hernandez Sanabria M, Liu Z, Ruvolo P, Thompson PA, Ferrajoli A, Daher M, Burger J, et al: The CXCR4-STAT3-IL-10 pathway controls the immunoregulatory function of chronic lymphocytic leukemia and is modulated by lenalidomide. Front Immunol 8: 1773, 2018.

30. He S,Liao G,Liu Y,Huang L, Kang M and Chen L: Overexpression of STAT3/pSTAT3 was associated with poor prognosis in gastric cancer: A meta-analysis. Int J Clin Exp Med 8: 20014-20023, 2015.

31. Carretero R, Wang E, Rodriguez AI, Reinboth J, Ascierto ML, Engle AM, Liu H, Camacho FM, Marincola FM, Garrido F, et al: Regression of melanoma metastases after immunotherapy is associated with activation of antigen presentation and interferonmediated rejection genes. Int J Cancer 131: 387-395, 2012.

32. Randhawa J, Ostojic A, Vrhovac R, Atallah E and Verstovsek S: Splenomegaly in myelofibrosis - new options for therapy and the therapeutic potential of Janus kinase 2 inhibitors. J Hematol Oncol 5: 43, 2012.

33. Mathé E, Nguyen GH, Funamizu N, He P, Moake M, Croce CM and Hussain SP: Inflammation regulates microRNA expression in cooperation with p53 and nitric oxide. Int J Cancer 131: 760-765, 2012. 